\title{
3D Fabrication of Fully Iron Magnetic Microrobots
}

\section{Journal Article}

\section{Author(s):}

Alcântara, Carlos C.J.; Kim, Sangwon; Lee, Sunkey; Jang, Bumjin; Thakolkaran, Prakash; Kim, Jin-Young; Choi, Hongsoo; Nelson, Bradley J.; Pané, Salvador

\section{Publication date:}

2019-04-18

\section{Permanent link:}

https://doi.org/10.3929/ethz-b-000329251

\section{Rights / license:}

In Copyright - Non-Commercial Use Permitted

\section{Originally published in:}

Small 15(16), https://doi.org/10.1002/smll.201805006 


\section{D Fabrication of Fully Iron Magnetic Microrobots}

Carlos C.J. Alcântara, Sangwon Kim*, Sunkey Lee, Bumjin Jang, Prakash Thakolkaran, JinYoung Kim, Hongsoo Choi, Bradley J. Nelson*, and Salvador Pané*

C.C.J. Alcântara, Dr. S. Kim, Dr. B. Jang, P. Thakolkaran, Prof. B.J. Nelson, Dr. S. Pané Institute of Robotics and Intelligent Systems

ETH Zurich, Zurich

CH-8092, Switzerland

E-mail: kimsa@ethz.ch; bnelson@ethz.ch; vidalp@ethz.ch

S. Lee, Dr. J.-Y. Kim, Prof. H. Choi, Prof. B.J. Nelson

Robotics Engineering Department

Daegu Gyeongbuk Institute of Science and Technology (DGIST)

711-873, Daegu, South Korea

Keywords: direct laser writing, template-assisted deposition, iron electrodeposition, magnetic microrobots, upstream motion

Biocompatibility and high responsiveness to magnetic fields are fundamental requisites to translate magnetic small-scale robots into clinical applications. The magnetic element iron exhibits the highest saturation magnetization and magnetic susceptibility while exhibiting excellent biocompatibility characteristics. Here, a process to reliably fabricate iron microrobots by means of template-assisted electrodeposition in $3 \mathrm{D}$ printed micromolds is presented. The 3D molds are fabricated using a modified two-photon absorption (TPA) configuration, which overcomes previous limitations such as the use of transparent substrates, low writing speeds, and limited depth of field. By optimizing the geometrical parameters of the 3D molds, metallic structures with complex features can be fabricated. Fe microrollers and microswimmers were realized that demonstrate motion at approximately 20 body lengths per second, perform 3D motion in viscous environments, and overcome higher flow velocities than those of "conventional 3D printed helical microswimmers". The cytotoxicity of these microrobots is assessed by culturing them with human colorectal cancer (HCT116) cells for four days, demonstrating their good biocompatibility characteristics. Finally, preliminary 


\section{WILEY-VCH}

results regarding the degradation of iron structures in simulated gastric acid liquid are provided.

\section{Introduction}

The development of magnetic small-scale robots is motivated by its prospective applications in minimally invasive surgery, drug delivery, biopsy, and diagnosis. ${ }^{[1-7]}$ To translate this technology into real clinical applications, small-scale robots must fulfill several requirements, such as the capability of moving in different types of liquids and terrains, overcoming obstacles, and trespassing mechanical barriers. Biocompatibility of the device is also a crucial aspect. Finding a material that can satisfy all these requirements is a challenge, and solutions usually come from combining several materials on the same platform. For example, microrobots made of photocurable hydrogels containing superparamagnetic nanoparticles are potential candidates. ${ }^{[1,8,9]}$ However, the limited amount of magnetic material that can be loaded in these structures significantly reduces their locomotion abilities, such as swimming upstream, overcoming gravity, and penetrating mechanical barriers in biologically relevant scenarios. Other approaches consist of protecting a potential cytotoxic material, such as nickel or cobalt, with biocompatible coatings. However, this approach requires retrieval from the body at the end of the treatment.

Iron is among the most abundant elements in the Earth's crust, and it is a crucial trace metal for life, e.g., for the correct functioning of several hemoproteins such as ferritins, transferritins, or catalase. Consequently, micro- and nanorobots made of metallic iron are optimal candidates not only in terms of magnetic responsiveness, but also regarding its biocompatibility characteristics. Recently, the biosafety, degradation and long-term influence of pure iron stents in the human body has been positively assessed. ${ }^{[10,11]}$

Widespread methods to fabricate small-scale robots are glancing angle deposition (GLAD), self-assembly, and direct laser writing (DLW), also known as two-photon polymerization. ${ }^{[6]}$ The first and second approaches employ conventional thin film deposition 


\section{WILEY-VCH}

and patterning strategies to achieve simple 3D shapes with nanoscale resolution, but they are limited to simple designs such as helices and overhangs. Alternatively, DLW works based on the two-photon polymerization of suitable photocurable polymers and, in principle, does not impose constraints on attainable geometries. Briefly, DLW uses an ultrashort pulsed laser, which is focused so that its intensity within the focal volume allows for the multiphoton absorption by the photopolymer. As a result of this interaction with the laser, the photopolymer experiences modifications in its physical or chemical properties ${ }^{[12]}$. By moving the laser (or the sample) in three dimensions, a 3D structure can be written. The photocurable polymer or photoresist is then exposed to a developer solution, which either removes those parts that have been exposed to the laser (in the case of a positive photoresist), or those that have been unexposed (negative photoresist).

To overcome material limitations associated with this technique, 3D template-assisted electrodeposition was recently introduced as a strategy to infuse arbitrarily shaped microstructures made of metals, alloys, ceramics or conductive polymers (or combinations of these). ${ }^{[13-16]}$ This method is an appealing approach to produce microscale robotic architectures as it offers the versatility of DLW in producing virtually any mold or template with the wide variety of materials that can be grown using electrodeposition. Note that there are other directwrite processing methods such as direct ink writing (DIW) $)^{[17]}$ and focused electron beam induced deposition (FEBID) ${ }^{[18,19]}$ that could also be used to directly fabricate micrometallic structures. However, while DIW is capable of high-printing speeds, its shortcomings include a limited set of demonstrated attainable geometries and a constrained selection of metallic materials. The latter is able of accomplishing complex 3D geometries with feature sizes in the nanometer scale and composed of metals with high purity. However, the available writing speeds are unfeasible for the fabrication of a large number of structures with dimensions ranging from at least a few micrometers to a few millimeters, while keeping high-resolution features. 


\section{WILEY-VCH}

Here, we report on a design strategy for batch producing Fe microrobots based on 3D template-assisted electrodeposition. The three-dimensional mold is fabricated using a modified two-photon absorption (TPA) configuration, which overcomes previous limitations such as the use of transparent substrates, low writing speeds, and limited depth-of-field. ${ }^{[13]}$ More specifically, a positive-tone photoresist is immersed in a refractive index-matching elastomer, which directly contacts the objective (Figure 1a and Figure S1, Supporting Information). The substrate interface can then be easily detected because of the strong optical mismatch between this surface and the photoresist. As the layers are written, the mechanical stage moves away from the sample, eliminating depth of field limitations and reducing artifacts arising from spherical aberration. An advantage of this technique is that there are no constraints on the choice of substrate. Particularly, highly conductive layers can be employed as working electrodes for electrodeposition. Previously, this technique was limited to the use of transparent conductive oxides, which are electrochemically unstable ${ }^{[20]}$ and possess low electrical conductivity. An additional implication of our method is the possibility of using functional interfaces such as flexible layers, semiconductors and biomaterials, which are mostly opaque.

\section{Fabrication of Iron 3D Microstructures}

\subsection{Photoresist Micromolds}

The micromolds were prepared using a commercial TPA setup (Photonic Professional GT, Nanoscribe $\mathrm{GmbH}$ ). The 3D trajectories are defined by slicing and hatching (Figure S1, Supporting Information) standard 3D computer-aided design (CAD) geometries to expose pre-defined regions of a positive-tone photoresist. After thoroughly rinsing the templates with DI water to remove the refractive matching-index polymer (Figure S2, Supporting Information), the sample was post-baked to complete the photoreaction and then developed using a 2.38\% tetramethylammonium hydroxide-based developer (AZ 726MIF). The resulting 3D channels were then filled with electrodeposited iron (Figure 1b). Finally, the polymer 


\section{WILEY-VCH}

mold is removed using an organic solvent, revealing freestanding metallic microstructures (Video S1, Supporting Information).

We identified the chemically amplified positive photoresist AZ IPS6050 (Merck Performance Materials $\mathrm{GmbH}$, purchased from MicroChemicals $\mathrm{GmbH}$ ) as most suitable for fabricating our micromolds because of its improved uniformity and chemical, mechanical, and thermal stabilities. We exposed these templates to several environments, including a combination of ultrasonication, electrodeposition in acidic electrolytes, high temperatures, and vacuum, after which we did not observe any template degradation. We coated thick layers of AZ IPS6050 on bare glass, indium-tin oxide (ITO), gold, nickel, copper, titanium and gold surfaces. The photoresist was highly adherent to each one of these substrates without the need of adhesion promoters. In a single coating, we obtained thicknesses ranging from $30 \mu \mathrm{m}$ to approximately $250 \mu \mathrm{m}$ on $3 \mathrm{~cm}$ diameter glass coverslips by adjusting the speed and duration of the spin-coating step.

By appropriately choosing the soft-baking parameters, the photoresist was completely free of bubbles and cracks. Additionally, the optical transparency of AZ IPS6050 facilitates the inspection of 3D molds when compared to standard diazonaphthoquinone (DNQ)-novolac photoresists (Figure S1, Supporting Information). Using AZ 726MIF we developed structures with path lengths ranging from $30 \mu \mathrm{m}$ to $400 \mu \mathrm{m}$ between 1 minute and 30 minutes, depending on the geometry, laser power, and filament thickness. Additionally, when developing the same structures for up to $2 \mathrm{~h}$ we did not notice any dark erosion. According to our results and preliminary tests with other photoresists (AZ9260, AZ40XT and AZ4260), the performance of AZ IPS6050 was superior in all relevant aspects. The use of this new series of photoresist can be beneficial for other applications requiring thick and high-aspect ratio structures with sub-micron resolution. Lastly, we were able to use writing speeds of up to $5000 \mu \mathrm{m} / \mathrm{s}$, which is about two orders of magnitude higher than previous reports using the same technique. 


\section{WILEY-VCH}

\subsection{Electrodeposition of Iron}

Iron is typically electrodeposited using sulfate, chloride or mixed sulfate-chloride electrolytes. ${ }^{[21]}$ A hot chloride bath, also known as Fischer-Langbein solution, was recently used to electroform Fe stents. ${ }^{[11]}$ Despite its good mechanical and chemical properties, the operating conditions of this electrolyte are incompatible with our micromolds. Alternatively, we evaluated a mixed ferrous sulfate-chloride electrolyte, introduced in 1943 by Schaffert and Gonser. ${ }^{[22]}$ One of the main advantages of this formulation is that it does not contain any toxic compound, and, therefore, it is environmentally friendly and safe for human handling. The two main electrochemical reactions occurring at the cathode during electrodeposition of iron from solutions containing ferrous salts are the reduction of Fe(II) into metallic iron (Equation 1) and hydrogen evolution (Equation 2):

$$
\begin{aligned}
& \mathrm{Fe}^{2+}(\text { aq. })+2 \mathrm{e}^{-} \rightarrow \mathrm{Fe}(\mathrm{s}) \\
& 2 \mathrm{H}^{+}(\text {aq. })+2 \mathrm{e}^{-} \rightarrow \mathrm{H}_{2}(\mathrm{~g})
\end{aligned}
$$

In general, hydrogen evolution should be minimized in order to obtain Fe deposits with good properties, which can be achieved by properly tuning the bath composition and the electrochemical parameters. When performing potentiostatic deposition (-0.95V vs $\mathrm{Ag} / \mathrm{AgCl})$, at $\mathrm{pH} 2$ and $45^{\circ} \mathrm{C}$, we obtained bright, adherent, uniform and oxidation resistant layers. Under these conditions, the deposition rate varied as a function of the filament thickness and the total path length of the 3D template but remained within the $100 \mu \mathrm{m} / \mathrm{h}$ range. We observed that the use of a higher $\mathrm{pH}$ or different electrochemical potentials resulted in porous, dark and loose deposits. We tried to overcome this narrow operating window by introducing additives such as saccharin, sodium-lauryl sulfate, sodium citrate, glycine and boric acid. However, these compounds either worsened or did not improve the quality of the electrodeposited material. We also noticed that it is important to use the solutions within $24 \mathrm{~h}$ after preparation. As the bath ages, it remains light green and transparent, but the deposited material becomes increasingly porous and less homogeneous. We tried to extend the lifetime of the electrolyte 


\section{WILEY-VCH}

by adding ascorbic acid, which acts as a chemical reducing agent. When using concentrations around $1 \mathrm{~g} / \mathrm{l}$, the bath quickly becomes light blue, indicating the effectiveness of this additive. However, iron could not be electrodeposited using the same conditions previously mentioned. Therefore, we conjecture that an optimal concentration of $\mathrm{Fe}^{3+}$ is critical, and that the longterm stability of our bath is ensured by carefully maintaining the concentration of this cation. Note that the mixed sulfate-chloride bath used in this work etches iron quickly. Therefore, the mold should be immediately removed from the solution and rinsed with DI water as soon as the electrochemical cell is turned off. In parallel, we also electrodeposited nickel structures using a standard nickel sulfamate bath ${ }^{[21]}$ (Figure S3-S4, Supporting Information).

\subsection{Geometrical Design}

One of the main challenges in electrodeposition is hydrogen evolution ${ }^{[23]}$, which can have several deleterious effects on the quality of the final structure, such as undesired porosity and embrittlement. Moreover, hydrogen bubbles can impede the growth of the material as they can coalesce and block the cavities in the micromolds. Here, we overcome this aspect by designing the micromold in a way that its geometry is segmented into several branches by positioning multiple outlets and multiple contact points to the substrate. This strategy not only allows for facilitating the release of gas formed during plating, but also minimizes photoresist development and plating time. Unlike previously reported configurations, which enabled the detection of the photoresist-air interface, our modified method allows for the accurate detection of the photoresist-substrate interface. Therefore, the region near the substrate can be precisely designed. In the case of a helical shape, contact points are placed at every minor groove and an outlet at every major groove (Figure 1b). In this way, the effective path length for photoresist development and electrochemical growth is limited to $\pi \mathrm{R}$, where $\mathrm{R}$ is the radius of the helix and is independent of the number of turns and total length (Figure S6-S7, Supporting Information). Adopting this strategy, we did not observe problems arising from channel clogging (Figure S5, Supporting Information), overplating, and underplating, 


\section{WILEY-VCH}

provided that the path length between consecutive diverging and merging points of parallel channels remains identical. An additional advantage of segmenting the structures in parallel paths is that it creates an area modulation, which translates into current peaks during electrodeposition (Figure 1c). As a result, helices with different pitch angles can be electroplated in parallel and complex structures such as fully metallic double helices can be achieved (Figure 1d and Figure S7, Supporting Information). These peaks are an accurate indication of the section being currently plated and can be used as a robust cut-off condition, which is independent of potential, filament thickness, shape, temperature, or any other parameter. In Figure 1e, we show that the fabricated microstructures can be mounted on a 30gauge needle, illustrating the concept of delivering micro-agents using tools such as syringes and catheters.

\section{Experiments}

\subsection{Chemical composition, degradability and biocompatibility}

To assess the chemical composition of our structures, microswimmers and microrollers were analyzed by EDX mappings (Figure 2a) and line-scans (Figure 2b), which confirmed the presence of iron. The oxygen atomic weight percentage is approximately $15 \%$, with a sharp increase to over $50 \%$ in the region where parallel paths merge. In this area, hydrogen evolution may be exacerbated due to a local increase in current density. The observed oxygen can be originated from the generation of an oxide layer on the surface due to contact of the structures with air, or the formation of entrapped oxyhydroxides of iron (II, III) during the electrodeposition process.

Preliminary degradation tests were also performed by immersing the iron microstructures in simulated gastric acid liquid. Microhelices were visibly degraded within 20 minutes (Figure 2c). The biocompatibility of our structures was confirmed by culturing human colorectal cancer (HCT116) cells on the microrobots. A cell suspension volume of 


\section{WILEY-VCH}

$100 \mu \mathrm{L}$ containing 50 cells $/ \mathrm{mL}$ was placed on the structures (Figure $2 \mathrm{~d}$ ). During the first day, two cells are visible near the Fe structures. After one day, the cells visibly migrate towards the metallic part, and attach to it within two days. After three days, we observed that the cells proliferated and remained attached to our microrobots.

It has been well established that live cells are distinguished by the presence of ubiquitous intracellular esterase activity as determined by the enzymatic conversion of the virtually non-fluorescent cell-permeant calcein AM to the intensely fluorescent calcein, which is well retained within live cells. A $1 \mu \mathrm{M}$ concentration of calcein-AM was added with an equal volume of cell culture medium into the wells containing HCT116 cells and the microrobot, and then incubated for $15 \mathrm{~min}$ at room temperature. Stained HCT116 cells on the microrobot were imaged through a GFP filter to measure the green fluorescence of live cells. After attachment (day 2) and proliferation (day 4), no signs of cell toxicity were observed (Figure 2e).

\subsection{Magnetic Characterization}

A Vibrating Sample Magnetometer (VSM) was used to characterize the magnetization of Fe microrobots. Iron structures still embedded in the photoresist template were mounted on a quartz shaft and placed between two electromagnets (Figure 3a). The corresponding SEM images shows the geometry of the structures used in the measurement. The total mass is then calculated based on the electrodeposited charge (Figure 3b). The VSM loops of the structures reveal a soft-magnetic behavior with coercivities around 35 Oe and a saturation magnetization $\left(\mathrm{M}_{\mathrm{s}}\right.$ ) of approximately $1.2 \mathrm{~T}$ (Figure $3 \mathrm{c}$ ), which corresponds to $57 \%$ of the $\mathrm{M}_{\mathrm{s}}$ of pure $\alpha$-Fe $(2.1 \mathrm{~T})^{[24]}$. Note that the saturation value of our structures was obtained considering a $100 \%$ efficiency during the electrodeposition step. Smaller efficiencies would result in higher magnetization values. Additionally, the oxygen content reported in the previous section may contribute to a decrease in the magnetization of our microrobots. Nevertheless, the values herein reported are $36 \%$ higher than magnetite $\left(\mathrm{Fe}_{3} \mathrm{O}_{4}\right), 67 \%$ higher than cobalt ferrite 


\section{WILEY-VCH}

$\left(\mathrm{CoFe}_{2} \mathrm{O}_{3}\right)$, and $117 \%$ higher than $\mathrm{Ni}^{[24]}$. In Figure $3 \mathrm{~d}$, we show that our structures can reach approximately $42 \%$ of the saturation magnetization at fields as low as $50 \mathrm{mT}$, confirming their high performance under low magnetic fields and compatibility with current magnetic manipulation systems.

\subsection{Velocity Tests}

Next, we performed velocity tests on helical microswimmers and microrollers using rotating magnetic fields generated by a commercial system (MFG-100, Magnebotix AG). Figure 4a shows a 5-turn helix with $25 \mu \mathrm{m}$ radius and $20 \mu \mathrm{m}$ pitch distance, which was able to swim at a maximum average speed of $42 \mu \mathrm{m} / \mathrm{s}$ when actuated in $100 \mathrm{cSt}$ silicone oil at $10 \mathrm{mT}$ and $7 \mathrm{~Hz}$ (Video S2, Supporting Information). Magnetic field magnitudes of approximately $5 \mathrm{mT}$ represented the threshold, below which no noticeable motion could be observed. In lower viscosity environments, the interaction with the substrate is unavoidable because of the high density of fully metallic iron structures, which generates considerable motion drift. To exploit this feature, we also designed scaffold-like microrollers, which consist of five circular rings with $25 \mu \mathrm{m}$ diameter. When actuated in the same conditions as the microswimmers, a maximum average speed of $25 \mu \mathrm{m} / \mathrm{s}$ was observed at $10 \mathrm{mT}$ and $5 \mathrm{~Hz}$ (Figure $4 \mathrm{~b}$ and Video S3, Supporting Information). When manipulated in a low viscosity liquid (isopropyl alcohol (IPA)), a maximum average velocity of ca. $500 \mu \mathrm{m} / \mathrm{s}$ at $10 \mathrm{mT}$ and $30 \mathrm{~Hz}$ was obtained (Figure 4c and Video S4, Supporting Information), which corresponds to about 20 body lengths per second.

\subsection{Overcoming flow and gravity}

The enhanced magnetic torque that can be generated by fully magnetic microstructures may enable their navigation in the human vasculature in regions subjected to high flow velocities. To prove the hypothesis that fully metallic Fe microhelices are more suitable for this kind of application than their metal-coated polymer counterparts, we performed upstream motion measurements using both types of structures in a microfluidic channel (Figure 5a), which 


\section{WILEY-VCH}

serves as a 3D printed model of blood vessels. The microfluidic device consists of a straight channel with an inlet and an outlet at its extremities, and a loading port situated along the centerline (Figure 5b). Additionally, posts are placed along the channel to facilitate the identification of the starting position of the microrobot and the measurement of its displacement. To validate the assumption of a higher magnetic torque due to enhanced magnetic volume, a polymer helix with the same dimensions (as its fully metallic counterpart) was coated with $20 \mathrm{~nm}$ Ti and $300 \mathrm{~nm}$ Ni by e-beam evaporation. The maximum thickness of the nickel layer before the metal starts to peel off is $300 \mathrm{~nm}$.

Using a commercial pump system (NE-300, New Era Pump Systems Inc.), the performance of the metal-coated and the fully metallic helices was compared under different flow velocities in water (Figure 5c and Video S5-S6, Supporting Information) while actuating them with a rotating magnetic field at $20 \mathrm{mT}$ and $30 \mathrm{~Hz}$. At $32 \mu \mathrm{m} / \mathrm{s}$, both structures managed to swim upstream, but the polymer structure showed an unstable behavior. Above this value, at $63 \mu \mathrm{m} / \mathrm{s}$, only the fully metallic structure could overcome the downstream flow. However, it was swept away when the magnetic field was turned off, thus indicating that its additional weight does not influence the measurement. Values around $95 \mu \mathrm{m} / \mathrm{s}$ represented the threshold above which the torque exerted by our Fe helices was insufficient to perform upstream motion.

Iron helices can also overcome gravity when actuated in $100 \mathrm{cSt}$ silicone oil. As shown in Figure 5d, the microhelix drifts when actuated near a solid surface. By applying an in-plane rotating magnetic field, the microhelix can move upwards, which is indicated by a loss of camera focus. To further confirm the out-of-plane displacement, we restore the magnetic field to its initial configuration. As a result, the microrobot moves forward without drift, confirming an offset from the substrate plane (Video S7, Supporting Information). 


\section{WILEY-VCH}

\section{Conclusion}

In summary, we fabricated iron microstructures using a robust and environmentally friendly process. The microrobots are biocompatible and exhibit high magnetic torque, thus enabling their use in biomedical applications. In addition to small-scale robotics, micro-sized metal parts are increasingly relevant for several other applications, such as biodegradable stents, load-bearing scaffolds for bone regeneration, 3D spintronic circuits, photonic metamaterials, and microparts for the aviation and the automotive industries. ${ }^{[1,15,17,25-27]}$ Our method can be extended to overcome challenges in the additive manufacturing of micrometallic structures with respect to feature size, attainable geometries, reproducibility, writing speeds, scalability, and choice of metals to be deposited. ${ }^{[17]}$

\section{Experimental Section}

Fabrication of 3D templates: AZ IPS6050 (from MicroChemicals GmbH) was first spincoated on a glass substrate at 2400 rpm for 18 seconds followed by a soft bake process using a hot plate at $125^{\circ} \mathrm{C}$ for 5 minutes with a heating ratio of approximately $15^{\circ} \mathrm{C}$ per minute. A drop of refractive-index matching oil (Immersol 518f, Carl Zeiss Microscopy GmbH) was placed on top of the photoresist and mounted on top of an oil-immersion $63 \mathrm{x}$ objective (numerical aperture $=1.4$ from Zeiss). Using the software DeScribe (Nanoscribe GmbH), the 3D geometries were sliced in multiple layers with a fixed distance of $0.3 \mu \mathrm{m}$ along the vertical direction. Each layer was then hatched in the X-Y plane in lines separated by $0.3 \mu \mathrm{m}$. The laser power and scan-speed were then set to $32 \%$ of the maximum power (at power scaling $=$ 1.0) and $2500 \mu \mathrm{m} / \mathrm{s}$, respectively. The sample is then exposed using a laser with a $780 \mathrm{~nm}$ wavelength and at room temperature. We limit the total exposure time to $24 \mathrm{~h}$, after which it becomes harder to completely remove Immersol $518 \mathrm{f}$ residues from the sample. After the exposure is complete, the sample is baked at $100^{\circ} \mathrm{C}$ for $140 \mathrm{~s}$. The substrate is finally developed in AZ 726MIF between 1 and 30 minutes and rinsed with DI water. 


\section{WILEY-VCH}

Fabrication of microfluidic channels: The microfluidic channel was fabricated using a conventional soft-lithography process. Firstly, a SU-8 master was fabricated on a 4-inch silicon wafer by photolithography. SU-8 2075 photoresist (Microchem Corp., USA) was spincoated on the wafer with $70 \mu \mathrm{m}$ thickness and exposed by UV (i-line with 365nm wavelength) through a transparency emulsion mask to define the microfluidic channel. Unexposed SU-8 was then selectively removed using SU-8 developer. The SU-8 mold was coated with trichloro(1H, 1H, 2H, 2H-perfluoroctyl)silane (Sigma-Aldrich, South Korea) using a vapor silanization procedure in order to reduce PDMS adhesion. Next, PDMS (Sylgard 184, Dow Corning, USA) mixed with its curing agent (10:1) was poured on the SU-8 master and cured at $80^{\circ} \mathrm{C}$ for $1 \mathrm{~h}$. The PDMS replica was then peeled off, and cut into individual microfluidic channel devices. Lastly, the surfaces of both the PDMS microfluidic channel and of a glass slide ( $25 \mathrm{~mm} \times 75 \mathrm{~mm}$ ) were treated with oxygen plasma (Femto Science, S. Korea), and then bonded together. In this manner, a PDMS microfluidic channel with $75 \mu \mathrm{m}$ width and $70 \mu \mathrm{m}$ height was formed and used to investigate the swimming of the developed microrobot against fluidic flow.

Electrodeposition and SEM/EDX imaging: Chemicals were purchased from Sigma-Aldrich and used as received. Electrodeposition was carried out using a three-electrode cell setup connected to a commercial potentiostat (PGSTAT204, Metrohm AG). A double junction $\mathrm{Ag} / \mathrm{AgCl}$ was used as the reference electrode and a $5 \mathrm{~cm} \times 5 \mathrm{~cm}$ flat platinum sheet as the counter electrode (CE). The distance between the chip with the template (working electrode) and the CE was fixed at approximately $0.5 \mathrm{~cm}$. The electrolyte was prepared with Millipore Milli-Q water and always used within $24 \mathrm{~h}$ after mixing $\mathrm{FeSO}_{4} .7 \mathrm{H}_{2} \mathrm{O}(250 \mathrm{~g} / \mathrm{L}), \mathrm{FeCl}_{2} .4 \mathrm{H}_{2} \mathrm{O}$ (42 g/L), and $\mathrm{NH}_{4} \mathrm{Cl}(20 \mathrm{~g} / \mathrm{L})$. The electrolyte was kept at constant temperature using a hot plate with a connected thermocouple immersed in the solution. The samples were deposited at $45^{\circ} \mathrm{C}$ and $\mathrm{pH} 2$ (adjusted with $\mathrm{H}_{2} \mathrm{SO}_{4}$ ) and deposited under constant potential (-0.95V vs $\mathrm{Ag} / \mathrm{AgCl})$ and under mild agitation using a $2.5-\mathrm{cm}$ magnetic stirring bar (100 rpm). The 


\section{WILEY-VCH}

volume of the electrolyte for the plating was fixed at $200 \mathrm{ml}$. The current onset was used as the cut-off condition. The samples were then immediately removed from the electrolyte and rinsed with DI water to avoid metal redissolution. The morphology of the structures was characterized by scanning electron microscopy (SEM) and their chemical composition was evaluated using energy dispersive X-ray spectroscopy (EDX) with a high-resolution fieldemission SEM (Leo 1530, Carl Zeiss AG).

Cell experiments: HCT116 cells were prepared at a concentration of 50 cells/ml in RPMI 1640 growth medium (Thermo Fisher Scientific, S. Korea) supplemented with $10 \%$ fetal bovine serum, $100 \mathrm{~g} / \mathrm{ml}$ penicillin, and $10 \mathrm{~g} / \mathrm{ml}$ streptomycin. Next, a single microrobot was added with $100 \mu \mathrm{L}$ of the medium into a well in a transparent 96-well plate with $\mathrm{U}$ shapedbottom coated with anti-adhesion layer (Thermo Fisher Scientific, S. Korea). Then, $100 \mu \mathrm{L}$ of the prepared cell suspension was additionally injected into the well. They were cultured together for 2 days to allow cells to adhere to the microrobot. After 2 days culturing, HCT116 cells were observed using an inverted microscope (Observer Z1, Carl Zeiss AG, Germany) with a 40x objective lens in bright-field mode. For determination of cell viability, live cells were stained with calcein AM (ex/em 488 nm/515 nm, Thermo Fisher Scientific, S. Korea).

\section{Supporting Information}

Supporting Information is available from the Wiley Online Library or from the author.

\section{Acknowledgements}

The authors thank Hen-Wei Huang, Ayoung Hong, and Daniel Ahmed for their comments and discussions, and David Sargent for his help with magnetic manipulation experiments. This project was financially supported by the Swiss Secretariat of Research and Innovation in the frame of the European Union's Horizon 2020 SELECTA project under the Marie Sklodowska-Curie Actions (H2020MSCA-ITN-2014, No. 642642). The authors acknowledge funding provided by the Robot Industry Fusion Core Technology development project through the Korea Evaluation Institute of Industrial Technology (KEIT) funded by the Ministry of Trade, Industry and Energy of Korea (MOTIE) (No. 10052980), and by the Global Research Laboratory (GRL) Program through the National Research Foundation of Korea (NRF) funded by the Ministry of Science and ICT (No. NRF-2017K1A1A2013237). 


\section{WILEY-VCH}

Received: ((will be filled in by the editorial staff))

Revised: ((will be filled in by the editorial staff)) Published online: ((will be filled in by the editorial staff))

References

[1] S. Tottori, L. Zhang, F. Qiu, K. K. Krawczyk, A. Franco-Obregõn, B. J. Nelson, Adv. Mater. 2012, 24, 811.

[2] S. Kim, F. Qiu, S. Kim, A. Ghanbari, C. Moon, L. Zhang, B. J. Nelson, H. Choi, Adv. Mater. 2013, 25, 5863.

[3] S. Fusco, M. S. Sakar, S. Kennedy, C. Peters, R. Bottani, F. Starsich, A. Mao, G. A. Sotiriou, S. Pané, S. E. Pratsinis, D. Mooney, B. J. Nelson, Adv. Mater. 2014, 26, 952.

[4] A. Servant, F. Qiu, M. Mazza, K. Kostarelos, B. J. Nelson, Adv. Mater. 2015, 27, 2981.

[5] C. Peters, M. Hoop, S. Pané, B. J. Nelson, C. Hierold, Adv. Mater. 2016, $28,533$.

[6] X.-Z. Chen, B. Jang, D. Ahmed, C. Hu, C. De Marco, M. Hoop, F. Mushtaq, B. J. Nelson, S. Pané, Adv. Mater. 2018, 30, 1705061.

[7] M. Sitti, Nature 2009, 458, 1121.

[8] F. Qiu, L. Zhang, K. E. Peyer, M. Casarosa, A. Franco-Obregón, H. Choi, B. J. Nelson, J. Mater. Chem. B 2014, 2, 357.

[9] X. Wang, X.-H. Qin, C. Hu, A. Terzopoulou, X.-Z. Chen, T.-Y. Huang, K. ManiuraWeber, S. Pané, B. J. Nelson, Adv. Funct. Mater. 2018, 1804107.

[10] M. Moravej, D. Mantovani, M. Moravej, D. Mantovani, Int. J. Mol. Sci. 2011, 12, 4250.

[11] M. Moravej, A. Purnama, M. Fiset, J. Couet, D. Mantovani, Acta Biomater. 2010, 6, 1843.

[12] N. Anscombe, Nat. Photonics 2010, 4, 22.

[13] M. A. Zeeshan, R. Grisch, E. Pellicer, K. M. Sivaraman, K. E. Peyer, J. Sort, B. Özkale, M. S. Sakar, B. J. Nelson, S. Pané, Small 2014, 10, 1284. 


\section{WILEY-VCH}

[14] X. Wendy Gu, J. R. Greer, Extrem. Mech. Lett. 2015, 2, 7.

[15] J. K. Gansel, M. Thiel, M. S. Rill, M. Decker, K. Bade, V. Saile, G. Von Freymann, S. Linden, M. Wegener, Science (80-. ). 2009, 325, 1513.

[16] W. Zhou, S. M. Kuebler, K. L. Braun, T. Yu, J. K. Cammack, C. K. Ober, J. W. Perry, S. R. Marder, Science 2002, 296, 1106.

[17] L. Hirt, A. Reiser, R. Spolenak, T. Zambelli, Adv. Mater. 2017, 29, 1604211.

[18] J. D. Fowlkes, R. Winkler, B. B. Lewis, A. Fernández-Pacheco, L. Skoric, D. SanzHernández, M. G. Stanford, E. Mutunga, P. D. Rack, H. Plank, ACS Appl. Nano Mater. 2018, 1,1028 .

[19] M. M. Shawrav, P. Taus, H. D. Wanzenboeck, M. Schinnerl, M. Stöger-Pollach, S. Schwarz, A. Steiger-Thirsfeld, E. Bertagnolli, Sci. Rep. 2016, 6, 34003.

[20] L. Liu, S. Yellinek, I. Valdinger, A. Donval, D. Mandler, Electrochim. Acta 2015, 176, 1374.

[21] M. Izaki, in Mod. Electroplat., John Wiley \& Sons, Inc., Hoboken, NJ, USA, 2011, pp. $309-326$.

[22] R. M. Schaffert, B. W. Gonser, Trans. Electrochem. Soc. 1943, 84, 319.

[23] L. Hirt, S. Ihle, Z. Pan, L. Dorwling-Carter, A. Reiser, J. M. Wheeler, R. Spolenak, J. Vörös, T. Zambelli, Adv. Mater. 2016, 28, 2311.

[24] J. I. Schwerdt, G. F. Goya, M. Pilar Calatayud, C. B. Herenu, P. C. Reggiani, R. G. Goya, Curr. Gene Ther. 2012, 12, 116.

[25] A. Vyatskikh, S. Delalande, A. Kudo, X. Zhang, C. M. Portela, J. R. Greer, Nat. Commun. 2018, 9, 593.

[26] A. Fernández-Pacheco, R. Streubel, O. Fruchart, R. Hertel, P. Fischer, R. P. Cowburn, Nat. Commun. 2017, 8, 15756.

[27] K. Alvarez, H. Nakajima, K. Alvarez, H. Nakajima, Materials (Basel). 2009, 2, 790. 


\section{WILEY-VCH}

a

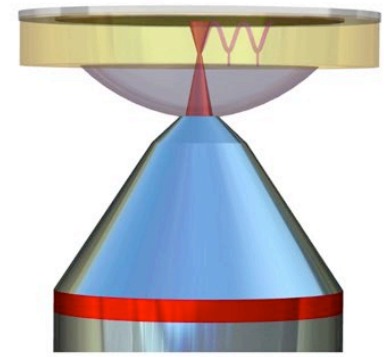

d

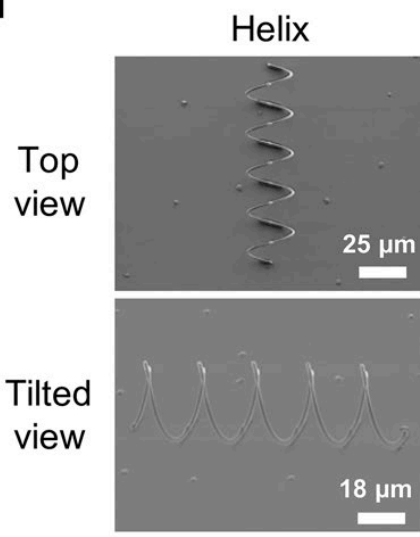

b

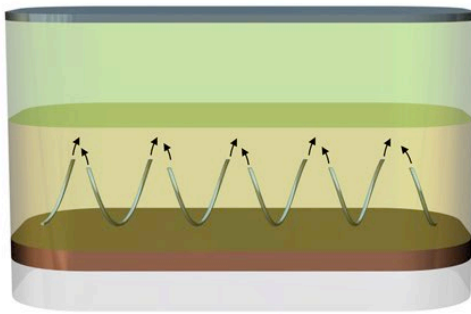

C

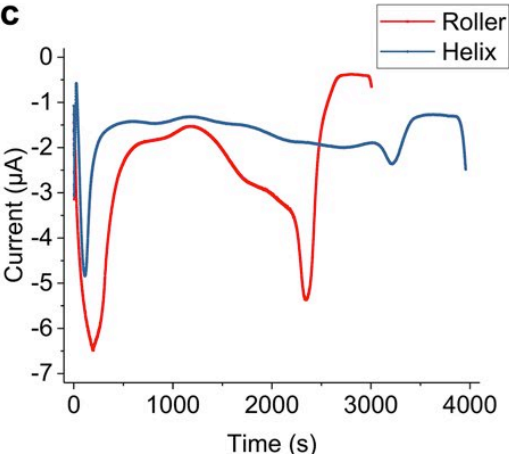

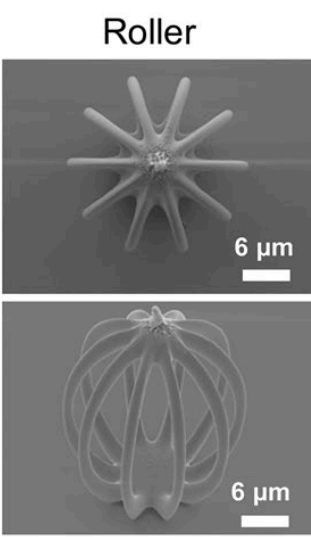
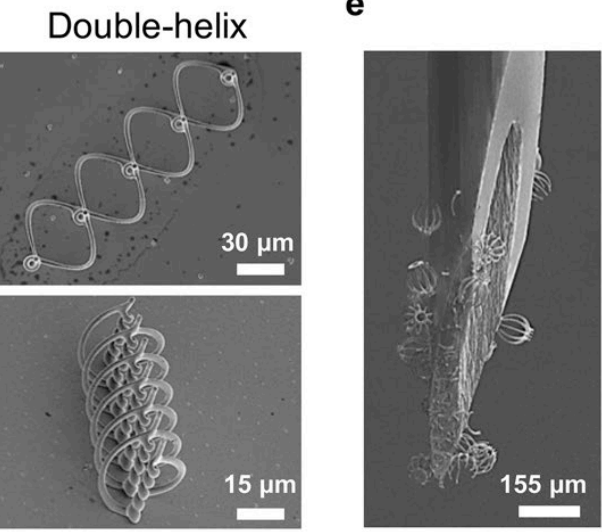

Figure 1. Fabrication of iron microstructures: (a) The metallic components are fabricated by 3D-assisted electrodeposition using a highly conductive and opaque substrate in a modified oil-immersion mode configuration, in which a chemically amplified positive-tone photoresist (AZ IPS6050) directly contacts the objective of a two-photon absorption (TPA) system, followed by electroplating and photoresist removal. (b) To electroplate complex mesoscale structures with features in the $1 \mu \mathrm{m}$ range, the geometry is segmented in several parallel segments, thus preventing bubble accumulation during electrodeposition and minimizing the plating time. (c) Finding a cut-off condition for the electrodeposition of 3D microstructures is difficult because of variations in the filament thickness, number of structures, temperature, metal salt concentration, $\mathrm{pH}$, etc. We realized that the geometry segmentation generates a current peak at every merging/diverging point, which serves as an accurate indication of the plating stage. (d) SEM images of micro-helices and micro-rollers, showing their highresolution features. (e) Microrobots mounted on a 30-gauge needle, illustrating the possibility of delivering microrobots using catheters and syringes. 


\section{WILEY-VCH}

a

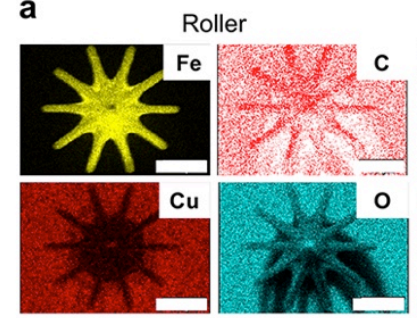

b

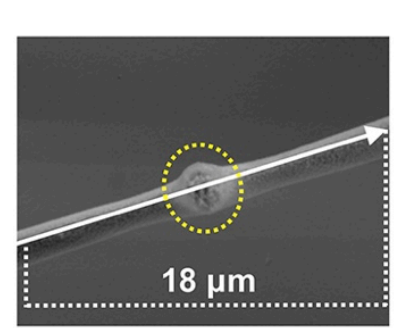

c

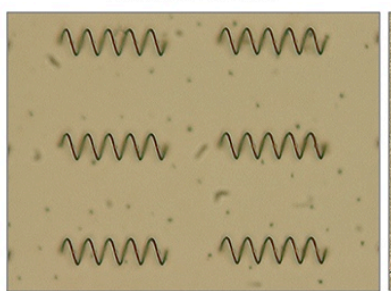

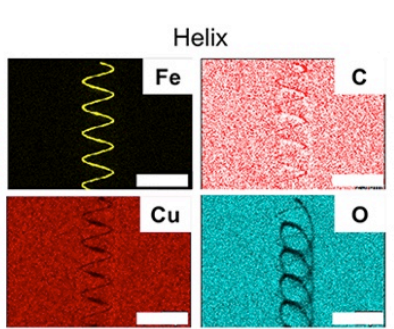

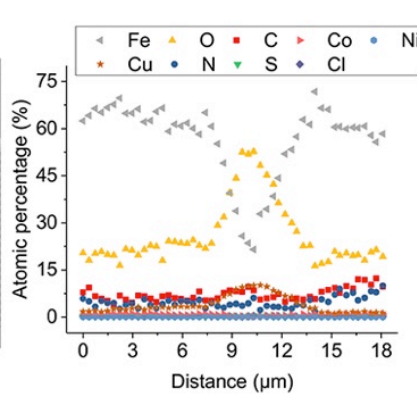

After 20 minutes

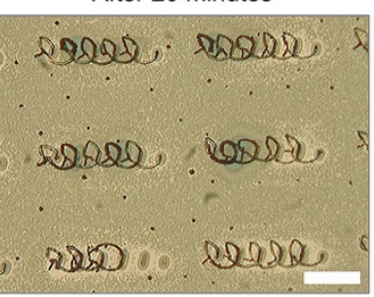

d

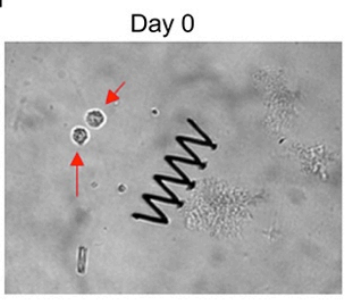

Day 2
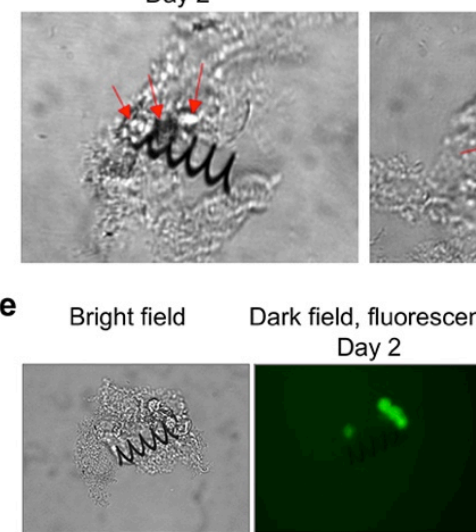

Day 4

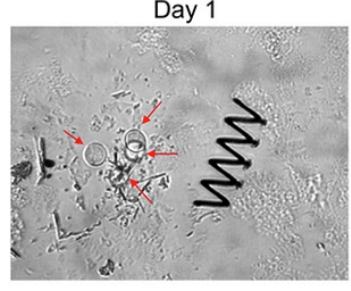

Day 3

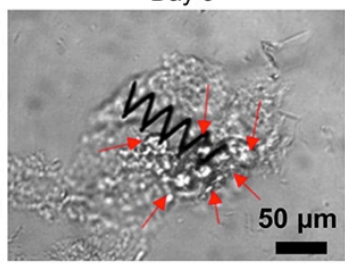

$50 \mu \mathrm{m}$
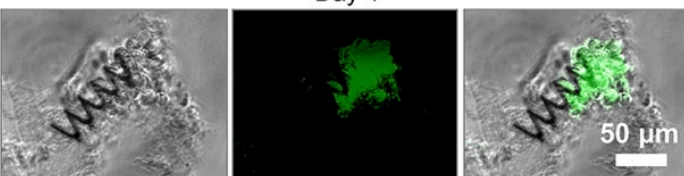

Figure 2. Chemical composition, degradability and biocompatibility of Fe microrobots: (a) EDX elemental mapping confirming the high intensity signal of iron and negligible influence of other elements. The scale bars are $10 \mu \mathrm{m}$ for the roller and $50 \mu \mathrm{m}$ for the helix. (b) A linescan along a merging point further confirms the purity of our structures and highlights an increased oxygen content in this junction, indicating the possibility of increased brittleness. (c) Microrobot degradation in simulated gastric liquid before and after 20 minutes. The scale bar is $50 \mu \mathrm{m}$. (d) Placement (day 0), migration (day 1), attachment (day 2) and proliferation (day 3) of human colorectal cancer (HCT116) cells cultured on the microrobots. (e) Bright field and fluorescent images of HCT116 cells attached to the microrobots after 2 days and 4 days. No signs of cytotoxicity were detected. 


\section{WILEY-VCH}

a
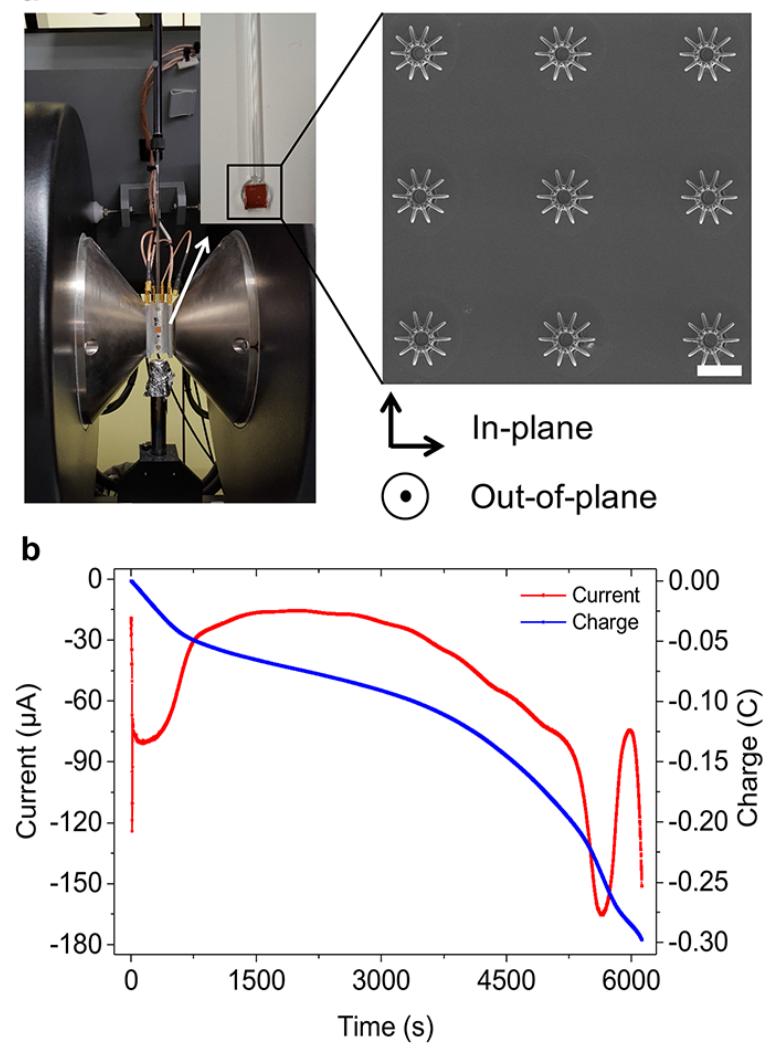

C
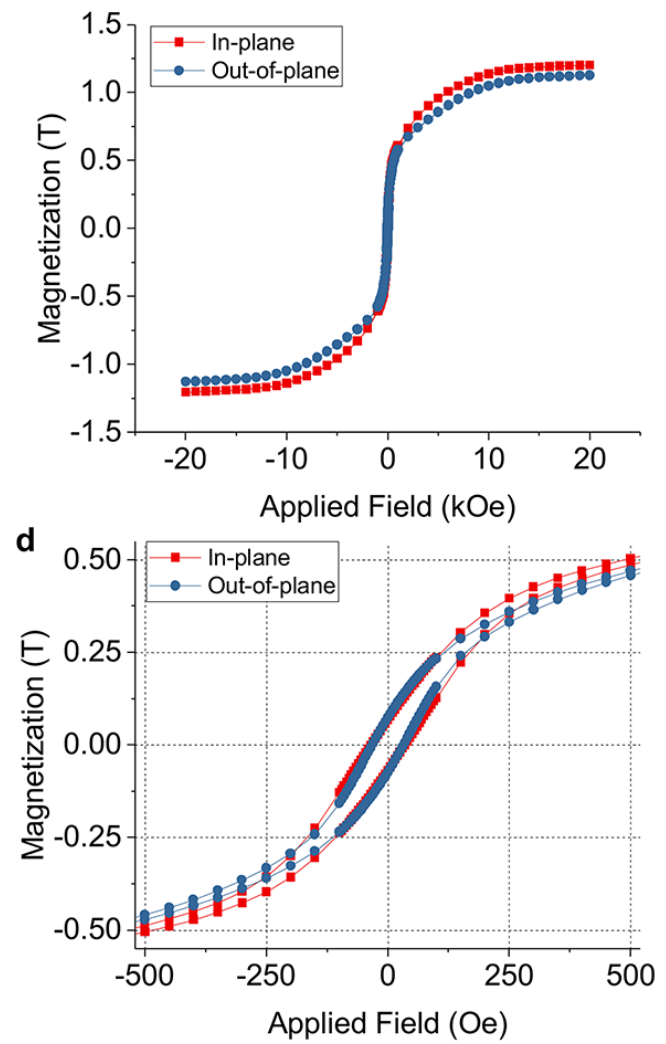

Figure 3. Magnetic characterization of Fe microstructures: (a) VSM setup used for the measurements, where structures embedded in a photoresist template are mounted on a quartz shaft and placed between two electromagnets. The SEM image shows the geometry of the $80 \mu \mathrm{m}$ metallic parts after dissolving the polymer mold. The scale bar is $80 \mu \mathrm{m}$. (b) The electrodeposited charge was used to calculate the mass using the Faraday's law of electrolysis. (c) In-plane and out-of-plane VSM loops obtained from an array of structures (as in a) showing a high saturation magnetization of $1.2 \mathrm{~T}$. (d) At $50 \mathrm{mT}$, a magnetization of 0.5 $\mathrm{T}$, approximately $42 \%$ of the saturation magnetization, can be achieved. 


\section{WILEY-VCH}

a

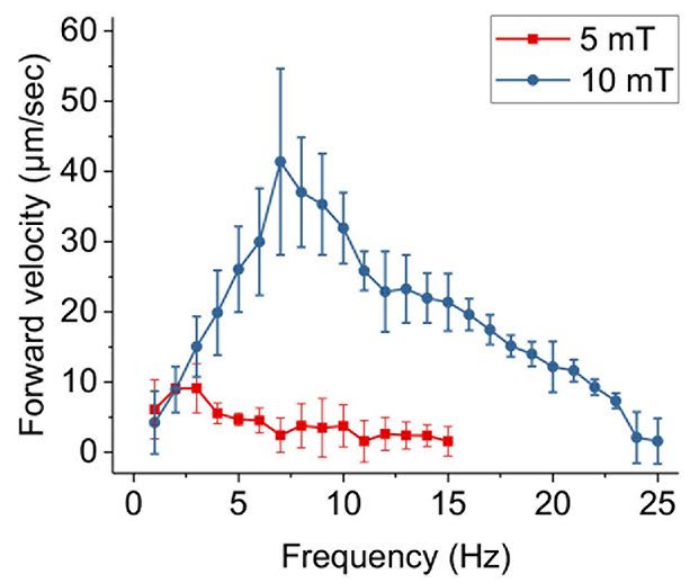

Microswimmer, $100 \mathrm{cSt}$, Silicone oil

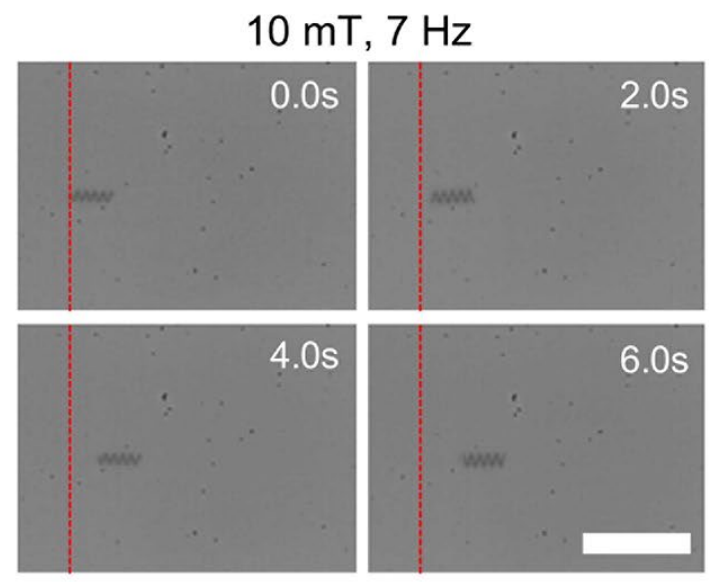

b

\section{Microroller, $100 \mathrm{cSt}$, Silicone oil}
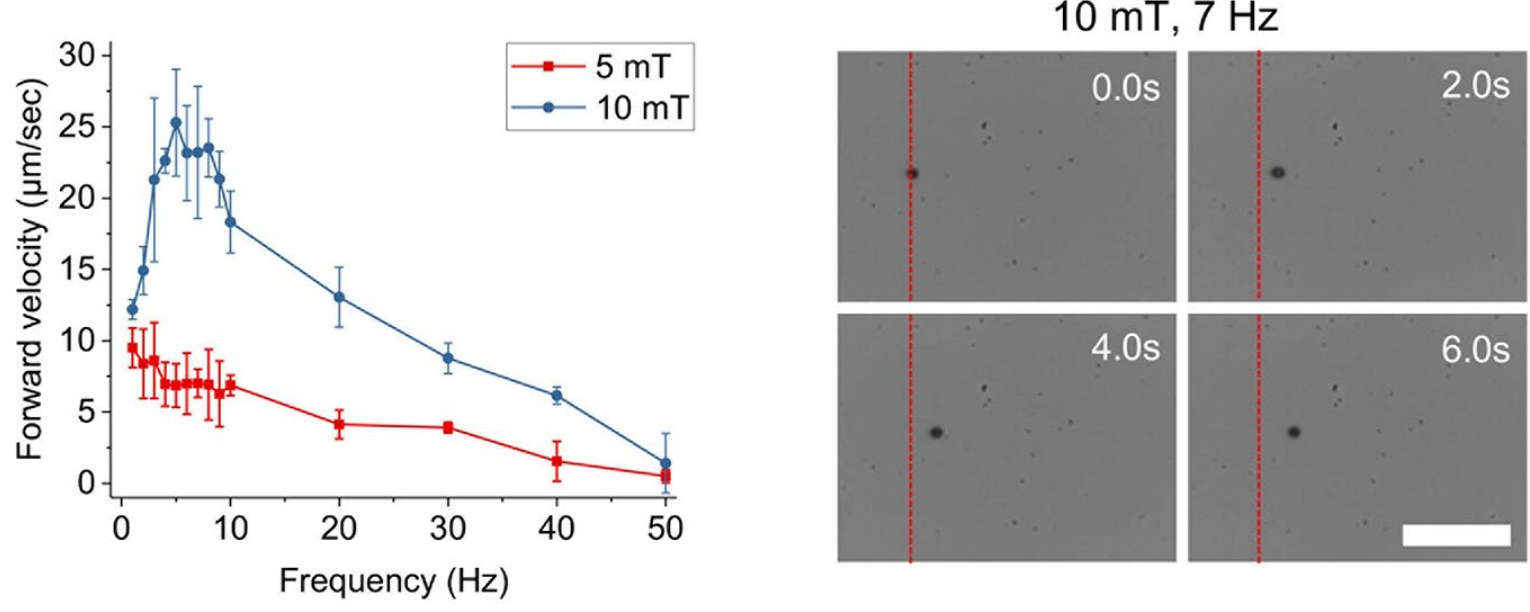

C

Microroller, 1 cSt, IPA

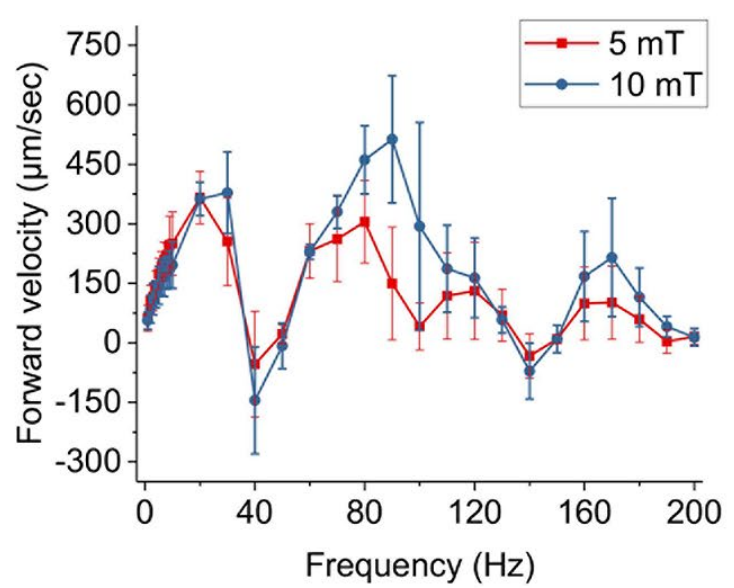

$10 \mathrm{mT}, 30 \mathrm{~Hz}$
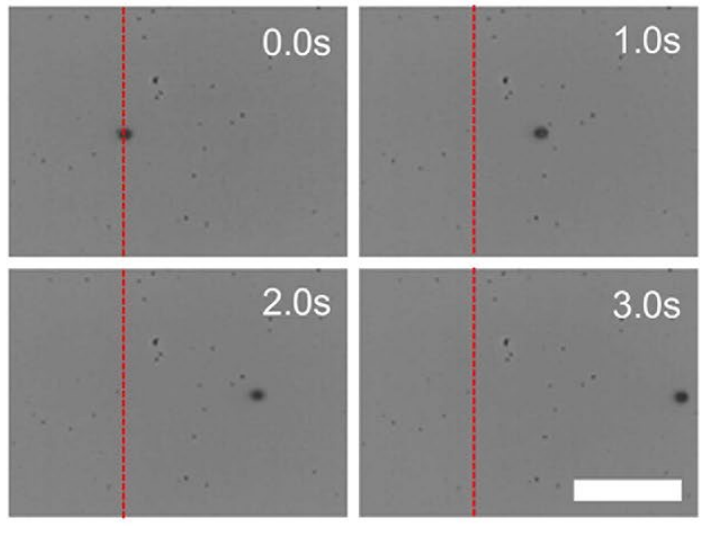

Figure 4. Velocity measurements of iron microrobots in liquids with different viscosities: (a) Because of its enhanced magnetic volume, iron microhelices can swim in $100 \mathrm{cSt}$ silicone oil at a maximum average speed of $42 \mu \mathrm{m} / \mathrm{s}$ when magnetically actuated at $10 \mathrm{mT}$ and $7 \mathrm{~Hz}$. (b) Under the same magnetic field and environment, micro-rollers can move at $25 \mu \mathrm{m} / \mathrm{s}$ at $7 \mathrm{~Hz}$. (c) As a result of its higher density, the same rollers can achieve peak velocities of $500 \mu \mathrm{m} / \mathrm{s}$ in low viscosity environments, such as isopropyl-alcohol (IPA). All the scale bars are $200 \mu \mathrm{m}$. 


\section{WILEY-VCH}

a

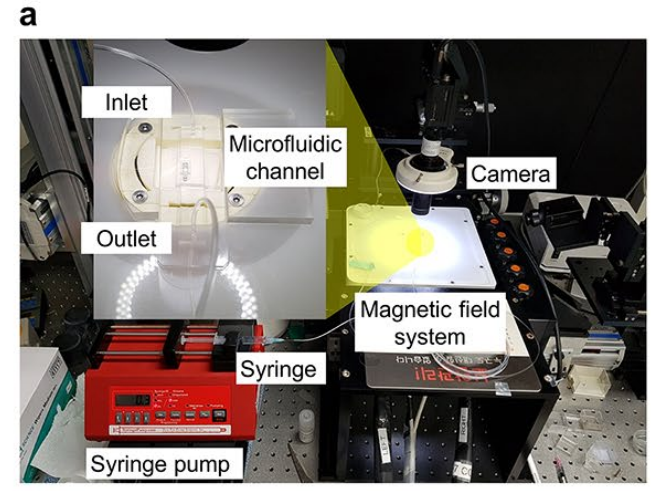

b

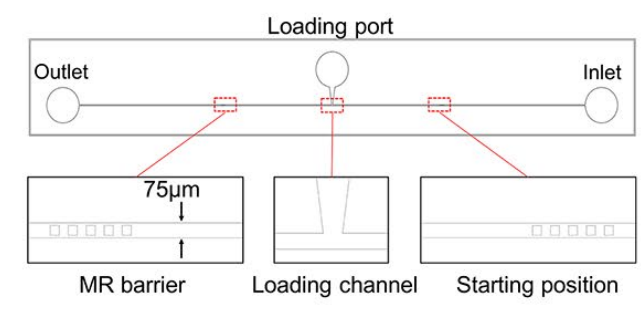

d

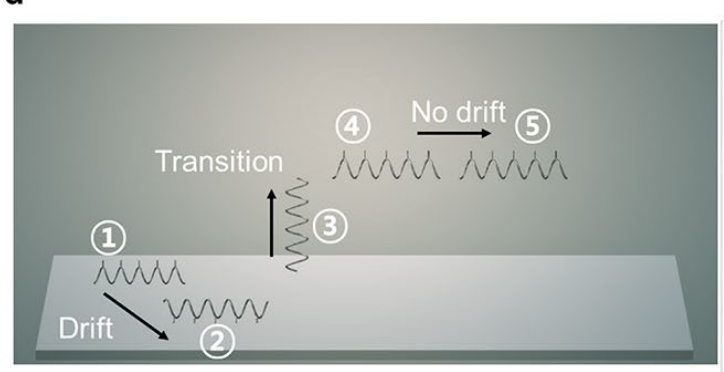

C

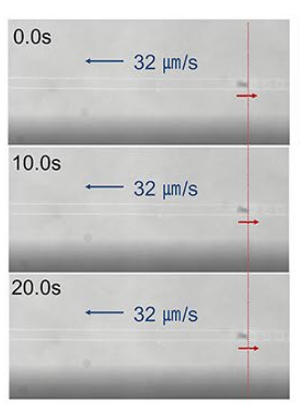

Nickel-coated $(300 \mathrm{~nm})$ polymer microrobots
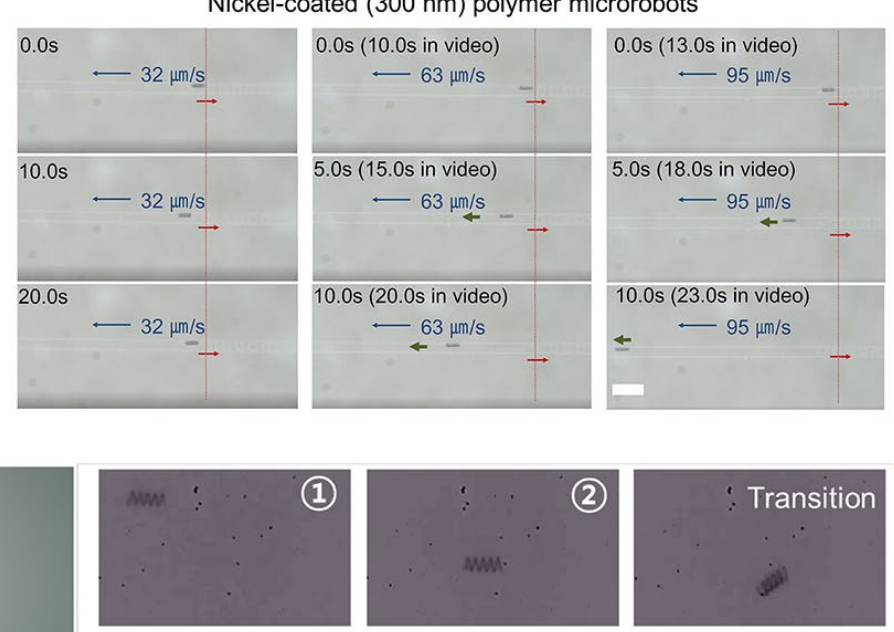

(3)
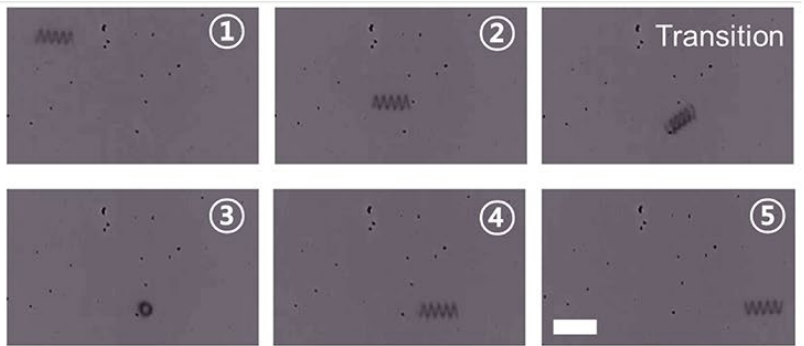

Fully Iron microrobots

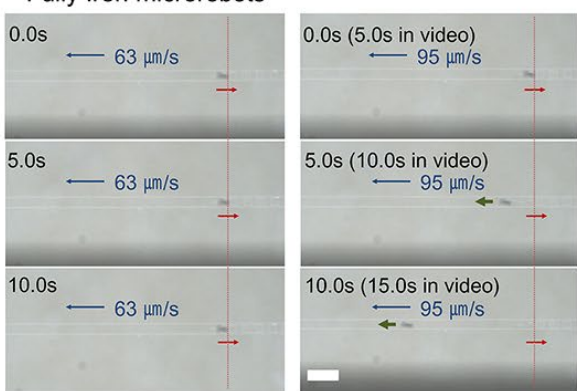

(4)

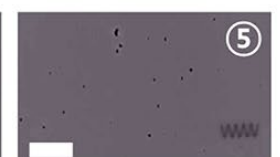

Figure 5. Overcoming flow velocities and gravity: (a) Setup to conduct upstream motion measurements against flow in water, where a microfluidic channel coupled to a syringe pump is mounted on a magnetic actuation system. (b) Microchannel fabricated using standard soft lithography. A straight line with $75 \mu \mathrm{m}$ width and $70 \mu \mathrm{m}$ height is coupled with its respective inlet, outlet, loading port and mechanical posts. (c) Performance comparison between a fully Fe microrobot and a polymer helical structure with the same dimensions and coated with 20 $\mathrm{nm} \mathrm{Ti}$ and $300 \mathrm{~nm} \mathrm{Ni}$. The blue arrows indicate the flow direction, while the red arrows indicate the magnetic field direction. The red vertical lines correspond to the starting position, when the pump is turned on $(t=0)$. Note that the videos were recorded before the pump was turned on. For this reason, we provide also the corresponding time in the movie. The fully magnetic structures can overcome higher flow velocities, up to ca. $95 \mu \mathrm{m} / \mathrm{s}$, highlighting the enhanced performance of the Fe microrobots. The scale bars are $200 \mu \mathrm{m}$. (d) Microhelices can overcome gravity in $100 \mathrm{cSt}$ silicone oil. In this experiment, the microrobot experiences motion drift because of its interaction with the substrate (steps 1 and 2). By directing the field upwards (transition step), the structure can overcome gravity (step 3) and, as the field direction is restored, swim forward without drift (steps 4 and 5). The scale bar is $100 \mu \mathrm{m}$. 